\title{
Testing Monotonicity of Pricing Kernels
}

\author{
Yuri Golubev* \\ Wolfgang Härdle** \\ Roman Timonfeev**
}

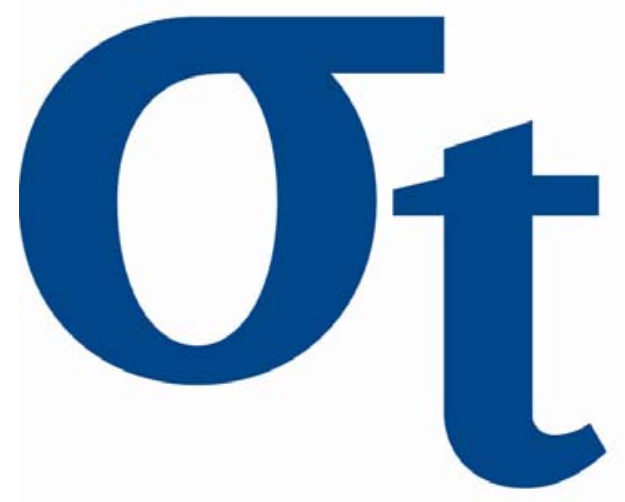

* CMI Université de Provence, France

** Humboldt-Universität zu Berlin, Germany 


\title{
Testing Monotonicity of Pricing Kernels *
}

\author{
Yuri Golubev \\ CMI, Universite de Provence \\ 39, rue F. Joliot-Curie, 13453 Marseille, Cedex 13, France \\ Wolfgang K. Härdle \\ CASE - Center for Applied Statistics and Economics \\ Humboldt-Universität zu Berlin, \\ Spandauer Straße 1, 10178 Berlin, Germany \\ Roman Timofeev \\ CASE - Center for Applied Statistics and Economics \\ Humboldt-Universität zu Berlin, \\ Spandauer Straße 1, 10178 Berlin, Germany
}

October 10, 2007

\footnotetext{
*We gratefully acknowledge financial support by the Deutsche Forschungsgemeinschaft and the Sonderforschungsbereich 649 "Ökonomisches Risiko". Roman Timofeev's research was supported by Deka Bank scholarship program.
} 


\begin{abstract}
The behaviour of market agents has always been extensively covered in the literature. Risk averse behaviour, described by von Neumann and Morgenstern (1944) via a concave utility function, is considered to be a cornerstone of classical economics. Agents prefer a fixed profit over uncertain choice with the same expected value, however lately there has been a lot of discussion about the reliability of this approach. Some authors have shown that there is a reference point where market utility functions are convex. In this paper we have constructed a test to verify uncertainty about the concavity of agents' utility function by testing the monotonicity of empirical pricing kernels (EPKs). A monotone decreasing EPK corresponds to a concave utility function while non-monotone decreasing EPK means non-averse pattern on one or more intervals of the utility function. We investigated the EPK for German DAX data for years 2000, 2002 and 2004 and found the evidence of non-concave utility functions: $H_{0}$ hypothesis of monotone decreasing pricing kernel was rejected at $5 \%$ and $10 \%$ significance level in 2002 and at $10 \%$ significance level in 2000.
\end{abstract}

JEL classification codes: G12, C12

Keywords: Risk Aversion, Pricing kernel 


\section{Introduction}

The behaviour of market agents has always been in focus in economic literature. Neumann and Morgenstern (1944) describe risk averse behaviour using concave utility functions. Agents prefer a fixed profit over uncertain choice with the same expected value, however lately there has been a lot of discussion about the reliability of this approach. Recent empirical studies by Jackwerth, J. C. (2002) showed that there is a reference point near the inital wealth where market utility functions are convex. Rosenberg, J. and Engle, R. (2002) observed a region of negative absolute risk aversion for orthogonal polynomial pricing kernel. Detlefsen, et al (2007) raised the same question by recovering utility function through empiricial pricing kernels for different time periods and observed a bump in EPK functions near zero returns.

In this paper we test the concavity of utility function by checking the monotonicity of pricing kernel. A strictly decreasing EPK corresponds to a concave utility function which is consistent with classical theory of risk averse behaviour, while rejection of monotone decreasing EPK would mean non-averse pattern of the utility function. By analysing empirical pricing kernels we can also identify on which interval or intervals monotonicity of EPK was rejected. This setup is consistent with the main goal of the paper to test for monotonicity in a particular region (e.g. near zero returns), although the results can be different on other intervals (e.g. for large positive and negative returns the behaviour of EPK can be unpredictable due to scarcity of the data).

In Figure 1.1 we compare utility functions obtained from DAX index in year 2000 and derived from Black and Scholes model. Black and Scholes model is equivalent to monotone increasing and concave utility function, see panel left of the figure. A nonparametric estimator that replicates option prices provides us with market utility function, depicted on the panel right of the figure. As can be observed, market utility function has a slight bump over the region of zero returns. The aim of this paper is to find out whether observed fluctuations are significant.

Construction of empricical pricing kernels has been well described by Ait-Sahalia and Lo (2000). In their paper they distinguish the concept of economical risk which contains 

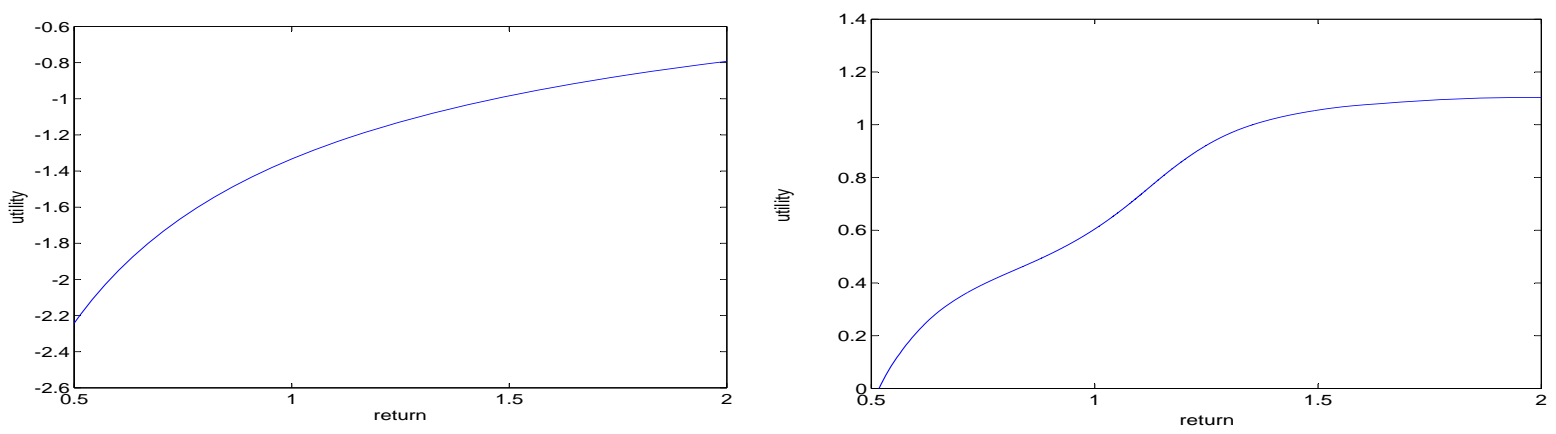

Figure 1.1: Classical utility function produced from Black Scholes model (left) and market utility function estimated from empirical pricing kernel on 06/30/2000 (right)

investors' preferences and statistical risk, which provides information on the risk of the data generating process. Economic risk is well approximated by Arrow-Debrue prices and can be estimated by risk neutral density $q$ obtained from the derivative market. Their work offers several accurate estimators of $q$ using, for example, Black and Scholes (1973) model and also present nonparametric estimators. In this paper risk neutral density is derived from implied volatility models combined with Heston model, see Detlefsen, et al (2007) for details. These kind of models provide a better fit because they incorporate sudden price jumps and explain volatility smile. Due to the large number of observations in derivative option market, risk neutral density $q$ can be precisely estimated and considered to be known. Statistical risk is presented by the distribution $p$ of future prices conditional on current prices. Some attempts to estimate $p$ were undertaken by Rosenberg, J. and Engle, R. (2002) using GARCH model and Ait-Sahalia and Lo (2000) using a nonparametric diffusion model. The main difficulty in estimating $p$ is that it depends on the underlying process of price $S_{t}$ and can only be estimated using the historical time series of $S_{t}$. Therefore, estimation of historical density $p$ is complicated by model specification and data scarcity and considered to be unknown. Thus, we would like to test monotonicity of pricing kernel constructed as a ratio of estimated $q$ and unknown $p$.

Ait-Sahalia and Lo (2000) in their paper offer another test for risk neutrality and specific preferences. Depending on the form of preferences they define $H_{0}$ hypothesis as a relationship between estimated neutral density $q$ and subjective density $p$. We do not make any assump- 
tions about the form of preferences and also consider subjective density $p$ as unknown. In our test $H_{0}$ hypothesis of monotone decreasing EPK is compared to a general class of all possible functions under $H_{1}$. The test is constructed as follows: first the spacing method is used to reduce the problem to an exponential model. On the basis of this model likelihood ratio test is applied for a fixed interval and using intersection of tests for different intervals it is expanded to a test independent of intervals. Finally, test statistics, calculated on observed data, is compared to simulated critical values and a final decision about monotonicity is taken.

The paper is organised as follows. In Section 2 we introduce important notations and problem setup which is then reduced to an exponential model using the spacing method. In Section 3 we formulate the hypotheses, construct a likelihood test for a fixed interval $[I, J]$ and then expand it to an independent test a using multiple testing technique. We also describe how to simulate critical values using the Monte-Carlo method. Section 4 contains the performance of the test for simulated data and Section 5 provides results on DAX data for 2000, 2002 and 2004.

\section{Conception of the Test}

\subsection{Problem Setup}

In this section we describe the relationships between (empirical) pricing kernel and utility function. Suppose we have at our disposal an i.i.d. sample of asset returns $X_{1}, \ldots, X_{n}$. Let $q(x)$ denote risk neutral density $Q(X<s)=\int_{-\infty}^{s} q(x) d x$ and $p(x)$ denote historical probability density $P(X<s)=\int_{-\infty}^{s} p(x) d x$ which is assumed to be unknown.

Consider an investor who optimizes his strategy by maximizing his utility function $U$ which gives us an asset equation:

$$
P_{t}=E^{P}\left[\beta \psi\left(X_{T}\right) \cdot \frac{U^{\prime}\left(X_{T}\right)}{U^{\prime}\left(X_{t}\right)}\right]
$$

where $\psi\left(X_{T}\right)$ is a payoff function and $E^{P}$ is the expectation with respect to the real/historical measure $\mathrm{P}$. 
Besides investor optimization problem, an asset can be priced under risk neutral measure $Q$ which enables us to construct a perfect hedge on derivative market:

$$
P_{t}=E^{Q}\left[\exp (-r T) \cdot \psi\left(X_{T}\right)\right]
$$

Defining pricing kernel $K(x)=\frac{q(x)}{p(x)}$ we derive:

$$
\begin{aligned}
P_{t}=\int_{-\infty}^{T} \exp (-r T) \psi\left(X_{T}\right) \cdot p(x) d x & =\int_{-\infty}^{T} \exp (-r T) \psi\left(X_{T}\right) \frac{q(x)}{p(x)} p(x) d x \\
& =E^{P}\left[\exp (-r T) \cdot \psi\left(X_{T}\right) \cdot K\left(X_{T}\right)\right]
\end{aligned}
$$

Combining both equations we obtain:

$$
\beta \frac{U^{\prime}\left(X_{T}\right)}{U^{\prime}\left(X_{t}\right)}=\exp (-r T) \cdot K\left(X_{T}\right)
$$

Equation (1) shows the relationship between utility function $U$ and pricing kernel $K$. Pricing kernel $K$ is proportional to marginal rate of substitution (MRS) between dates t and T. Therefore, the form of utility function up to a constant is defined by a pricing kernel as follows:

$$
U\left(X_{T}\right)=\frac{1}{\beta} U^{\prime}\left(X_{t}\right) \cdot \exp (-r T) \int_{-\infty}^{T} K(x) d x
$$

We want to test concavity of $U(x)$ by checking the monotonicity of $K(x)$. A strictly decreasing $K(x)$ corresponds to a concave utility function. We would like to check if there exists an interval $[a, b]$, where $K(x)$ is not monotone decreasing.

Denote by $X_{(1)}, \ldots X_{(n)}$ the order statistics related to $X_{1}, \ldots, X_{n}$ i.e.

$$
X_{(1)} \leq X_{(2)}, \ldots, \leq X_{(n)}
$$

With these notations we can rephrase our problem as follows: find (if possible) integers $I, J$ such that the sequence

$$
K_{k}=K\left(X_{(k)}\right)=\frac{q\left(X_{(k)}\right)}{p\left(X_{(k)}\right)}, \quad I \leq k \leq J
$$

is not monotone decreasing. The principal difficulty in this procedure is related to the fact that $p$ is considered to be unknown. To overcome this we will use three basic ingredients: 
- spacing method to reduce the problem to a simple exponential model

- maximum likelihood test to check monotonicity of $K_{k}$ for given $I$ and $J$

- multiple-testing procedure to find $I$ and $J$ on the basis of the data at hand.

\subsection{The Spacing Method}

Our method is based on the Pyke's theorem about the distribution of order statistics, see Pyke, R. (1965). Consider $U_{1}, \ldots, U_{n}$ be i.i.d with a uniform distribution on [0,1]. For the order statistics

$$
U_{(1)} \leq U_{(2)}, \ldots, \leq U_{(n)}
$$

define uniform spacings $S_{k}$ as

$$
S_{k}=U_{(k+1)}-U_{(k)} \text { and } S_{n}=U_{(n)}
$$

Theorem 2.1. Let $U_{1}, \ldots, U_{n}$ be i.i.d. uniformly distributed on $[0,1]$ and $e_{1}, \ldots, e_{n}$ be i.i.d. standard exponentially distributed random variables. Then

$$
\mathcal{L}\left\{S_{k}, 1 \leq k \leq n\right\}=\mathcal{L}\left\{\frac{e_{k}}{\sum_{i=1}^{n} e_{k}}, 1 \leq k \leq n\right\}
$$

Using the fact that $E\left[e_{k}\right]=1$ we obtain the following result:

$$
n\left\{U_{(k+1)}-U_{(k)}\right\}=n \cdot S_{k} \approx e_{k} .
$$

Let $P(x)=\int_{-\infty}^{x} p(u) d u$ be the probability distribution function associated with $p(x)$. Using $U_{(k)}=P\left(X_{(k)}\right)$ and first order Taylor approximation

$$
P\left(X_{(k+1)}\right)=P\left(X_{(k)}\right)+P^{\prime}\left(X_{(k)}\right) \cdot\left(X_{(k+1)}-X_{(k)}\right)
$$


we derive

$$
U_{(k+1)}-U_{(k)}=P\left(X_{(k+1)}\right)-P\left(X_{(k)}\right) \approx p\left(X_{(k)}\right) \cdot\left(X_{(k+1)}-X_{(k)}\right)
$$

Combining equations (4) with (3) we obtain

$$
n\left\{X_{(k+1)}-X_{(k)}\right\} q\left(X_{(k)}\right) \approx \frac{q\left(X_{(k)}\right)}{p\left(X_{(k)}\right)} e_{k}=K_{k} \cdot e_{k} .
$$

Thus our problem is reduced to the following one: check monotonicity of $K\left(X_{(k)}\right)=K_{k}$ using

$$
Z_{k}=K_{k} \cdot e_{k}, \quad I \leq k \leq J
$$

\section{Construction of the Test}

\subsection{ML test for given $I, J$}

Let $A(I, J)$ be the set of all possible decreasing sequences on a given interval $[I, J]$ :

$$
A(I, J)=\left\{a_{k} \geq a_{k+1}, I \leq k<J\right\}
$$

Let us defing the following hypotheses:

Hypothesis $H_{0}: \quad K \subset A(I, J)$ and pricing kernel $K$ is a monotone decreasing function Hypothesis $H_{1}$ : $K$ is any kind of function.

A nested model of monotone decreasing function under $H_{0}$ is compared to a general class of all possible functions under $H_{1}$ by calculating the maximum of likelihood function for each of the models. If function $K$ is non-monotone in accordance with $H_{1}$, maximum likelihood of two models should significantly differ from each other. On the other hand when we remove restriction on monotonicity and it does not bring significant improvement in likelihood, restricted model $H_{0}$ should be accepted.

The likelihood ratio monotonicity test is defined by the function

$$
\phi(Z)=\mathbf{1}\left\{\frac{\max _{K \subset A(I, J)}\{p(Z, K)\}}{\max _{K}\{p(Z, K)\}}-H_{\alpha}(I, J) \geq 0\right\}
$$


In other words, if $\phi(Z)=1$ we accept the null hypothesis $H_{0}: K \in A(I, J)$, otherwise the alternative is accepted. This setup can be simplified with the following monotone transformation:

$$
\phi(Z)=\mathbf{1}\left\{\log \frac{\max _{K \subset A(I, J)}\{p(Z, K)\}}{\max _{K}\{p(Z, K)\}}-h_{\alpha}(I, J) \geq 0\right\}
$$

For a given probability of the first kind error $\alpha$, the critical value $h_{\alpha}(I, J)=\log H_{\alpha}(I, J)$ is defined as root of the equation:

$$
\mathbf{P}_{0}\left\{\log \frac{\max _{K \in A(I, J)} p(Z, K)}{\max _{K} p(Z, K)}-h_{\alpha}(I, J) \leq 0\right\}=\alpha,
$$

where $\mathbf{P}_{0}$ is the probability measure generated by the observations from (5) with $K_{k} \equiv$ $1, I \leq k<J$.

Computation of $\max _{K} \log \{p(Z, K)\}$ is straightforward. Using the results from equation (5) that $Z_{k}=K_{k} \cdot e_{k}$ we derive log-likelihood function

$$
\log \{p(Z, K)\}=-\sum_{k=I}^{J} \frac{Z_{k}}{K_{k}}-\sum_{k=I}^{J} \log \left(K_{k}\right)
$$

which gives us analytical result for $\max _{K} \log \{p(Z, K)\}$ at $K_{k}=Z_{k}$ :

$$
\max _{K} \log \{p(Z, K)\}=-(J-I)-\sum_{k=I}^{J} \log \left(Z_{k}\right)
$$

Computation of $\max _{K \subset A(I, J)} \log \{p(Z, K)\}$ is performed via Newton-Raphson method with the projection on decreasing sequence $A(I, J)$. The main idea of this approach is to find the maximum likelihood over all possible monotone decreasing sequences by interative optimization via the Newton Raphson algorithm. This result is achieved through isotonic regression combined with Newton-Raphson opimization algorithm.

Isotonic regression performs the least square estimation subject to monotonicity contraint with strictly decreasing trend. For a given vectors $x, y$ of size $n$ the following minimization problem is fulfilled:

$$
\min _{f_{\text {iso }}} \sum_{i=1}^{n}\left\{y_{i}-f_{\text {iso }}\left(x_{i}\right)\right\}^{2} \text { s.t. } f_{\text {iso }}\left(x_{i}\right) \leq f_{\text {iso }}\left(x_{j}\right) \text { where } i>j
$$


where $f_{i s o}$ is isotonic regression. In practice isotonic regression represents a downward stepwise function, see Figure 3.1. This procedure is unfortunately very time-consuming. It can be also shown that $\max _{K \subset A(I, J)} \log \{p(Z, K)\}$ is obtained at isotonic regression over $Z_{k}$ parameters since $Z_{k}$ gives us $\max _{K} \log \{p(Z, K)\}$. Thus Newton-Raphson algorithm can be omitted, instead isotonic regression $f_{i s o}\left(Z_{k}\right)$ is applied to known $Z_{k}$.

$$
\max _{K \subset A(I, J)} \log \{p(Z, K)\}=-\sum_{k=I}^{J} \frac{Z_{k}}{f_{\text {iso }}\left(Z_{k}\right)}-\sum_{k=I}^{J} \log \left(f_{\text {iso }}\left(Z_{k}\right)\right)
$$

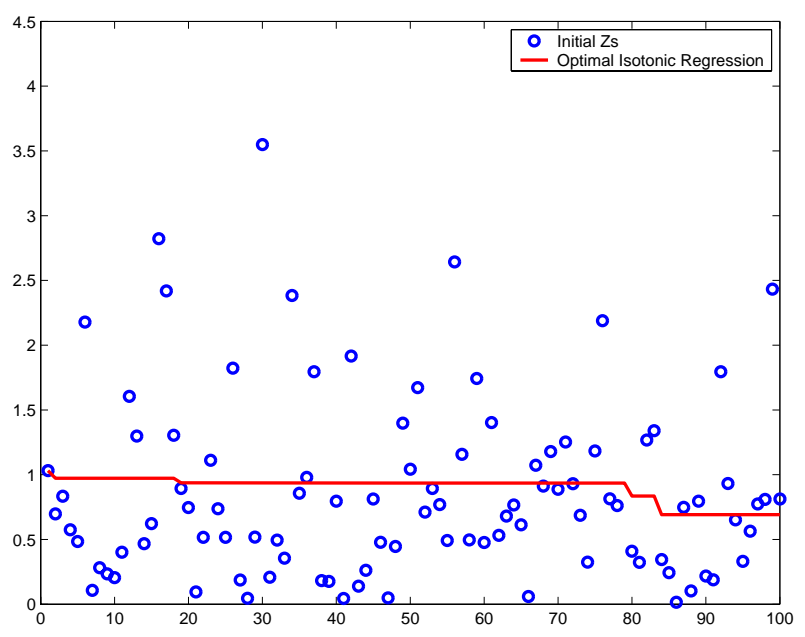

Figure 3.1: Isotonic Regression over $Z_{k}$ generated as iid standard exponential

\subsection{Multiple-testing}

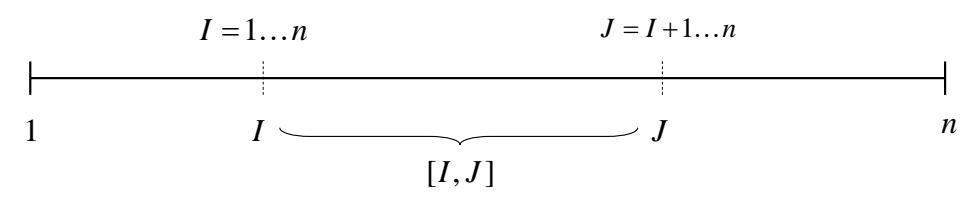

Figure 3.2: Multiple testing on intervals $I, J$

The principal idea in the multiple testing is to construct a test that does not depend on $I$ and $J$. This problem is typically solved with the help of tests intersection, see Berger 
(1982). The hypothesis $H_{0}$ of monotone decreasing function is rejected if it is rejected at least on one of the interval $[I, J]$, see Figure 3.2. It means that we are looking for a minimal critical surface $h(I, J)$ such that:

$$
\mathbf{P}_{0}\left\{\min _{I, J}\left\{\log \frac{\max _{K \in A(I, J)} p(Z, r)}{\max _{K} p(Z, K)}-h_{\alpha}(I, J)\right\} \leq 0\right\}=\alpha
$$

Unfortunately the exact solution of this problem is extremely difficult and unknown. Therefore we use the Monte-Carlo simulations to find a reasonable critical surface. We generate "the worst" non-increasing case of the sequence $K_{(k)}$ as a constant:

$$
K_{(1)}=K_{(2)}=\ldots=K_{(n)}=1
$$

Then using the result that $Z_{k}=K_{k} \cdot e_{k}$ we generate $Z_{k} \approx \exp (1)$ as an iid standard exponential random variable.

Let us define $\xi(I, J)$ as a test statistics over simulated $Z_{k}$ :

$$
\xi(I, J)=\log \frac{\max _{K \in A(I, J)} p(Z, K)}{\max _{K} p(Z, K)}=\max _{K \in A(I, J)} \log \{p(Z, K)\}-\max _{K} \log \{p(Z, K)\}
$$

Here $\xi$ is a matrix of dimensions $I, J$ with non-positive values. Maximum of value 0 is reached at any monotone decreasing interval $I, J$.

Define mean $M(I, J)$ and variance $V^{2}(I, J)$ of test statistics $\xi(I, J)$ :

$$
\begin{gathered}
M(I, J)=E_{0} \xi(I, J) \\
V^{2}(I, J)=E_{0}\left\{\xi^{2}(I, J)-E_{0} \xi(I, J)\right\}^{2}
\end{gathered}
$$

Parameters $M(I, J)$ and $V(I, J)$ are calculated by Monte-Carlo simulations of $Z_{k}$ as specified above.

Critical value $t_{\alpha}$, where $\alpha$ is a significance level, is calculated as a root of:

$$
P_{0}\left\{\min _{I, J}\left\{\xi(I, J)-M(I, J)+t_{\alpha} V(I, J)\right\} \leq 0\right\}=\alpha
$$


Equation (8) gives us a corresponding critical surface $h_{\alpha}(I, J)$

$$
h_{\alpha}(I, J)=M(I, J)-t_{\alpha} \cdot V(I, J)
$$

In Figure 3.2 the calculation algorithm of critical values $t_{\alpha}$ is displayed. Over all MonteCarlo simulations, $Z_{k}$ should violate $\alpha$-threshold surfaces $M(I, J)-t_{\alpha} \cdot V(I, J)$ in $\alpha$ percent cases.

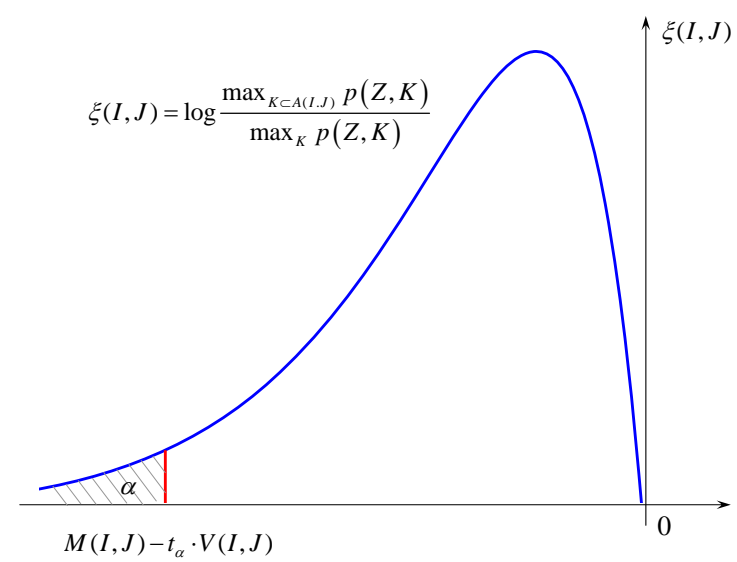

Figure 3.3: Calculation of critical value $t_{\alpha}$

\subsection{Multiple testing on blocks}

Suppose initial set of $Z_{k}$ can be divided in $m$ blocks of size $b$ and the remainder $n-b \cdot m$, see Figure 3.3 .

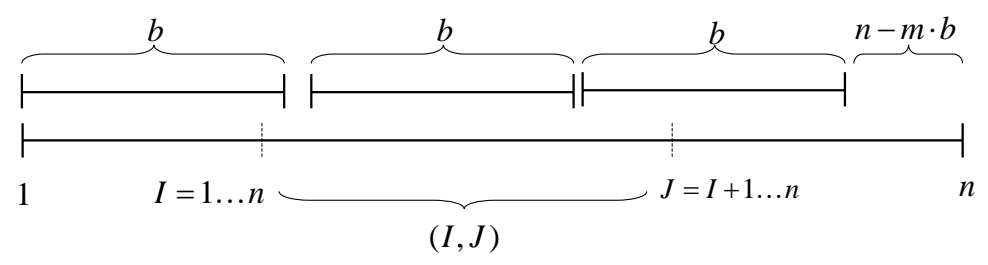

Figure 3.4: Multiple testing on blocks 
The idea to introduce blocks is motivated by the variance reduction. Initially we imply that the alternative hypothesis $H_{1}$ is a set of all possible functions. By introducing blocks we allow the function to be monotone decreasing on interval of size $b$ and thus we decrease the variance of the distribution. Blocks can be considered as a trade off between the variance reduction and shift parameter. For small size block distribution is shifted, but variance is also big. For large blocks the distribution function is less shifted but at the same time associated with smaller variance.

On the left panel of Figure 3.5 distribuction functions of test statistics without block and after introduction of block are depicted. First data are generated as linear trend with slope $b$, constant $a$ and iid exponential errors $e_{i}$ as $x_{i}=(a+b i) \cdot e_{i}$. Test statistics is obtained from equation (7) then ordered. Shift of distribution function is caused by increase of linear slope $b$ from 0 trend to 0.05 . This idea is an underlying principle of the test, non-monotone data shifts the ditribution to the left that should be determined by the test. Right panel of the figure shows the influence of block parameter on variance and shift of cdfs. At best we would like to maximize the shift and minimize the variance, with an increase of block size $m$ both shift and variance of cdf are smaller. The idea of blocks is to test monotonicity not only on each interval $I, J$ but also for all possible block sizes $b$.
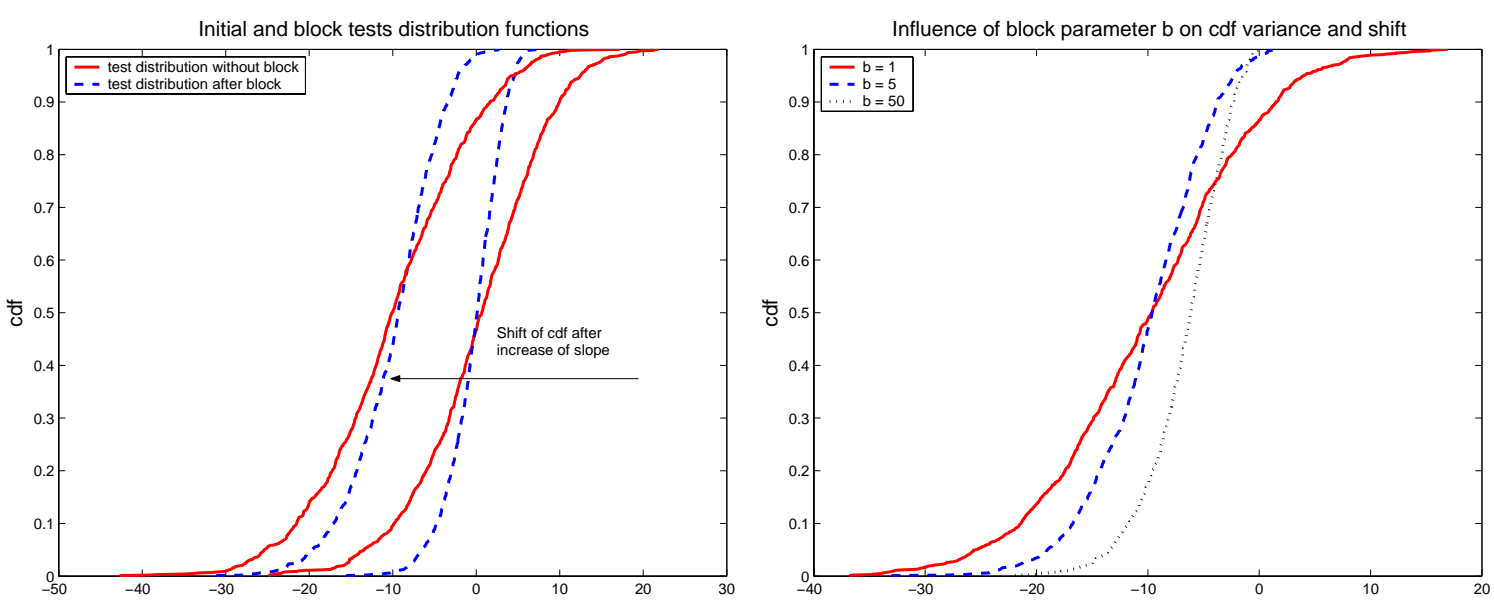

Figure 3.5: Multiple testing on blocks

Test statistics $\xi(I, J, b)$ is obtained as a difference between $\max _{K \in A(I, J)} \log \{p(Z, K)\}$ and $\max _{K} \log \{p(Z, K)\}$ but this time we assume that under $H_{1}$ function is monotone decreasing 
on each of $m$ blocks of size $b$. Instead of taking each value, average for each block is taken:

$$
\max _{K} \log \{p(Z, K)\}=-m-\sum_{j=1}^{m} \log \left(\frac{\sum_{k=j \cdot b-b+1}^{j \cdot b} Z_{k}}{b}\right)
$$

The same procedure is performed for calculation of $\max _{K \in A(I, J)} \log \{p(Z, K)\}$ but instead of $Z_{k}$ best monotone decreasing approximation is taken as an isotonic regression $f_{i s o}\left(Z_{k}\right)$.

Finally we can formulate hypotheses: $H_{0}$ hypothesis about monotonic decreasing function is rejected when monotonicity is rejected at least on one of the intervals $I, J$ with any block size $b$ :

$$
\min _{(I, J, b)}\left\{\xi(I, J, b)-M(I, J, b)+t_{\alpha, b} \cdot V(I, J, b)\right\} \leq 0
$$

Critical value $t_{\alpha, b}$ is different for each value of $b$ and obtained from the equation

$$
P_{0}\left\{\min _{I, J, b}\left\{\xi(I, J, b)-M(I, J, b)+t_{\alpha, b} \cdot V(I, J, b)\right\} \geq 0\right\}=\alpha
$$

Now we are ready to summarize the monotonicity test:

1. Compute $Z\left(X_{(k)}\right)=n \cdot q\left(X_{(k)}\right) \cdot\left\{X_{(k+1)}-X_{(k)}\right\}$

2. Compute test statistics

$$
\xi(I, J, b)=\log \frac{\max _{K \in A(I, J)} p(Z, K)}{\max _{K} p(Z, K)}=\max _{K \in A(I, J)} \log \{p(Z, K)\}-\max _{K} \log \{p(Z, K)\}
$$

3. Take decision: if

$$
\min _{I, J, b}\left\{\xi(I, J, b)-M(I, J, b)+t_{\alpha, b} \cdot V(I, J, b)\right\} \leq 0
$$

then $K(\cdot)$ is a non-monotone decreasing function 


\section{Implementation}

In this section the performance of monotonicity test for artifically simulated data is evaluated. We investigate the behavior of the test for different cases: monotone decreasing data, positive linear trend and sudden jumps. Simulated data are generated in accordance with one of the cases multiplied by standard exponential errors $e_{i}$. By simulating different errors we can obtain distribution function and then, basing on true function, calculate error probability and evaluate the power of the test.

Before we apply the test to simulated and observed data, important parameters have to be set. The decision about monotonicity is taken basing on sequence of surfaces $\xi(I, J, b)-$ $M(I, J, b)+t_{\alpha, b} \cdot V(I, J, b)$, one surface for each block size $b$. If at least one surface crosses zero level $H_{0}$ hypothesis about monotone decreasing funcion is rejected. If surface is located under zero level it means that calculated test statistics is to the left of threshold value $M(I, J, b)-t_{\alpha, b} \cdot V(I, J, b)$, see Figure 3.2. First we set the minimum interval of 10 observations between $J$ and $I$. This parameter is introduced to approximate test statistics $\xi$ with Gaussian distribution and improve the correlation betweeb statistics $\xi\left(I_{1}, J_{1}\right)$ and $\xi\left(I_{2}, J_{2}\right)$. Gaussian approximation is possible due to central limit theorem, the bigger the interval is, the better approximation. Obviously if the approximation is good, critical values $t_{\alpha}$ should be close to Gaussian critical values. Final goal of this parameter is to improve the power of the test.

The importance of $b$ parameter has been discussed in Section 3.3. Large $b$ reduces variance but at the same time decreases shift of the distribution. We start with value $b=1$ which corresponds to no block until $b=0.5 \cdot n$ which means the dataset is divided into exactly two blocks. Values more than $50 \%$ of observations would correspond to only one block and remainder and therefore do not make sense.

Calculation of critical values is described in Section 3.2. This procedure is very time consuming that is why we use dichotomic method in order to find the root of equation (8). This is a method of iterative splitting of intervals into halfs until required precision of solution is found.

First we generate a monotone decreasing sequence and check the performance of the test on this dataset. The "worst" monotone sequence is a constant therefore we simulate $x_{1}=x_{2}=$ 
$\cdots=x_{i}=1$. On the left panel of Figure 4.1 generated sequence $x, Z$ and corresponding isotonic regression over $Z_{i}$ are displayed. Having fixed $b=3$ we calculated critical values $t_{\alpha, 3}$ from equation (8) and corresponding testing surface $M(I, J, 3)-t_{\alpha, 3} \cdot V(I, J, 3)-\xi(I, J, 3)$ which are depicted on the right side of the figure.
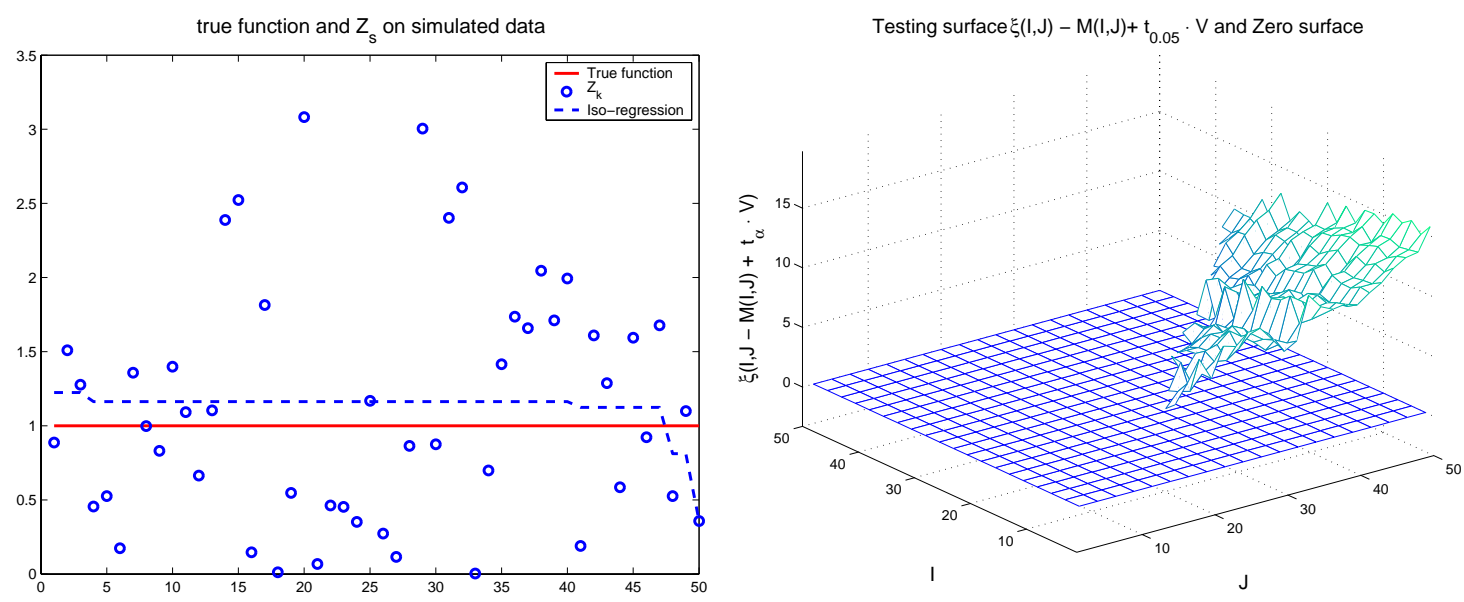

Figure 4.1: Simulated monotone data and resulting testing surface, $b=3$

The entire surface is located above zero level and therefore $H_{0}$ hypothesis of monotone decreasing function can not be rejected at $5 \%$ sifnificance level. The depicted above surface is a single result of generated errors $e_{i}$ and fixed parameter $b$ and therefore can not reflect overall performance of the test. In order to demonstrate overall behavior of the test we estimate error probability by generating different errors $e_{i}$. In Figure 4.2 distribution of first type error for different parameter $b$ is plotted, i.e. probability to accept $H_{1}$ although data are distributed under $H_{0}$. As it can be seen $b$ parameters does not improve the first type error.

In the next case data are generated with a positive linear trend $x_{i}=(a+0.05 \cdot i) \cdot e$, where $a$ is a constant and $i$ is an index from 1 to $n$. Simulated parameters $M(I, J, b)$ and $V(I, J, b)$ do no depend on data but only on parameters $b$ and number of observations $n$ and therefore can be taken from previously simulated example. For fixed $b=3$ generated data, rejection intervals and resulting surface $\xi(I, J, 3)-M(I, J, 3)+t_{\alpha, 3} \cdot V(I, J, 3)$ are given in Figure 4.3 . Rejection invervals show such $I$ and $J$ where testing surface crossed the zero level and $H_{0}$ was rejected. 


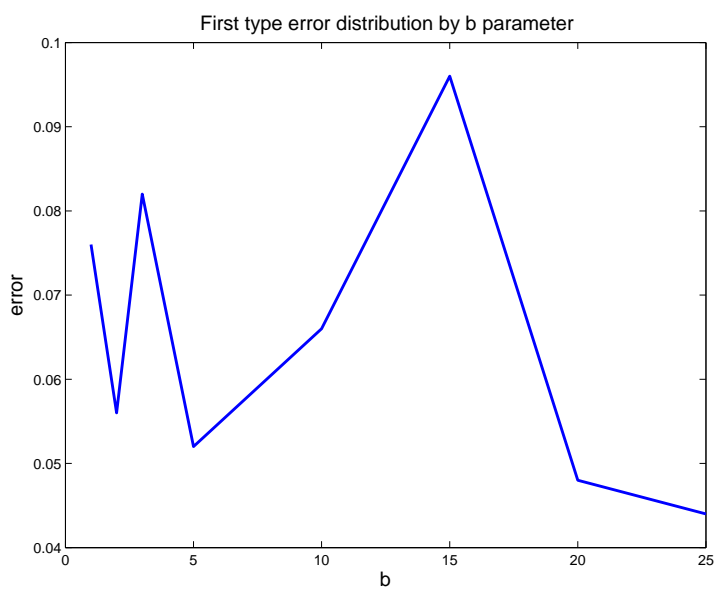

Figure 4.2: First type error distribution for different block parameter $\mathrm{b}$
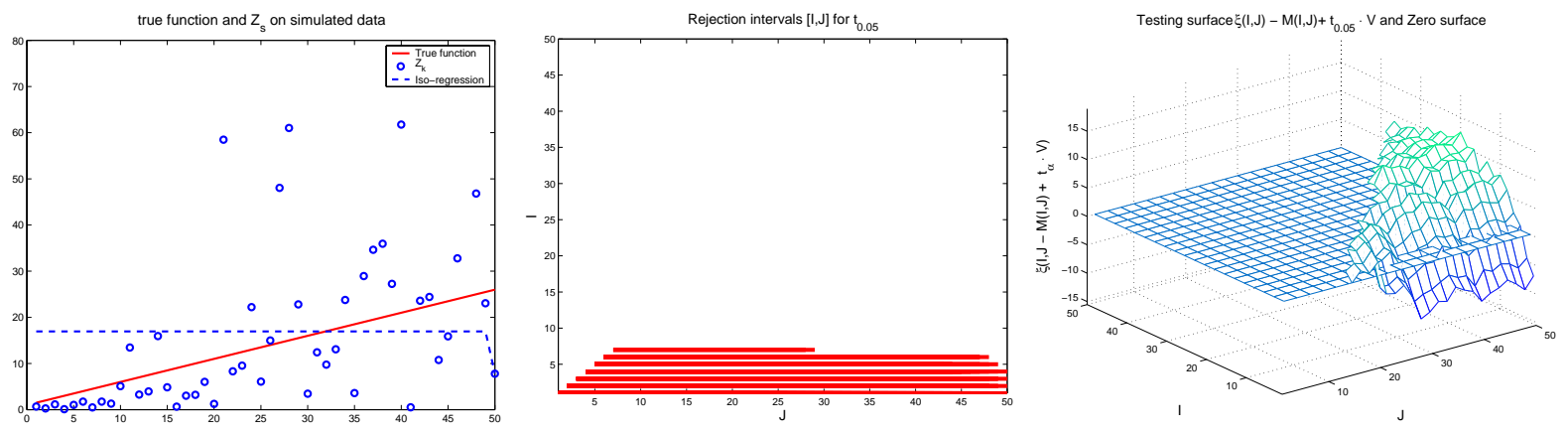

Figure 4.3: Simulated increasing data and resulting rejection intervals and testing surface

In order to calculate the error probability we calculate number of cases when test failed to identify non-monone structure of the data, i.e. second type error. On Figure 4.4 there is a distribution of second type erros for different $b$, starting from no block $(b=1)$ to exactly two intervals $b=0.5 \cdot n=25$. This figure shows that introduction of block significantly improves the performance of the test: error probability decreases from $75 \%$ for no block to almost $10 \%$ for $b=15$.

In next example we simulate an artificial bump, see left panel of Figure4.5. Ability of the test to identify jumps or bumps in pricing kernel function is especially important since observed data do not usually have an obvious positive trend. Instead EPK has various fluctuations, 


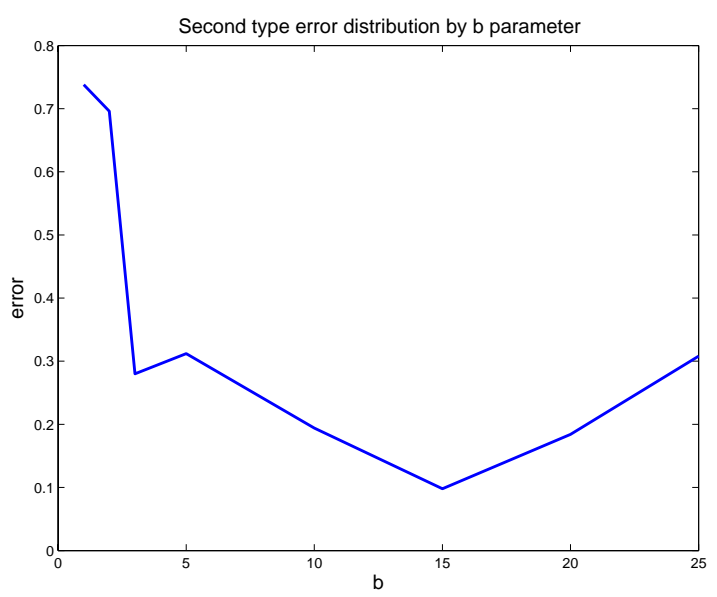

Figure 4.4: Second type error distribution for different block parameter $\mathrm{b}$

bumps and jumps. Significant bump would correspond to non-concave utility function and contradict to classical theory about risk-averse agents. On middle and right panels of Figure 4.5 testing surface $\xi(I, J)-M(I, J)+t_{\alpha} \cdot V(I, J)$ and rejection invervals $I, J$ are given for fixed block size $b=3$.
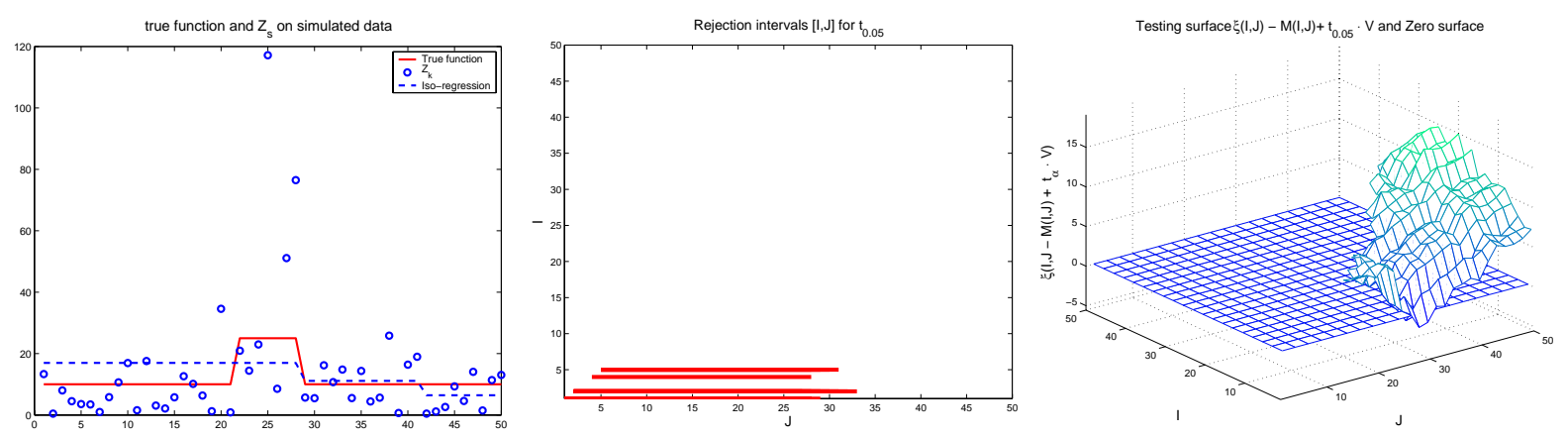

Figure 4.5: Simulated data with a bump and resulting rejection intervals and testing surface

Distribution of second type errors for different $b$ is given on Figure 4.6. We can see that there exists an optimal block size $b$ which corresponds to a trade off between shift and variance of distribution, see Section 3.3. Optimal $b$ is different for each dataset and therefore we consider a sequence of surfaces $\xi(I, J, b)-M(I, J, b)+t_{\alpha, b} \cdot V(I, J, b)$ for each block size $b$. $H_{0}$ hypothesis of monotonic decreasing function is rejected when at least one of these 
surfaces crosses zero level.

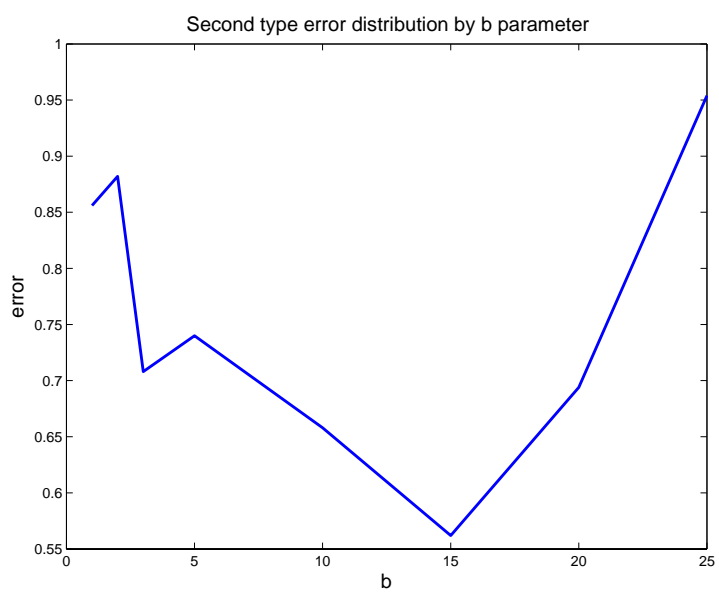

Figure 4.6: Second type error distribution for different block parameter b

\section{Monotonicity of DAX Empirical Pricing Kernel}

Final goal of this paper is to test empirical pricing kernel obtained from observed data. For the analysis we take data used in Detlefsen, et al (2007) where the pricing kernels and the risk aversion are analyzed in summer of years 2000, 2002 and 2004 in order to consider different market regimes. According to our test design the decision about monotonicity of pricing kernel is made on the basis of generated $Z_{k}=n \cdot\left(X_{(k+1)}-X_{(k)}\right) \cdot q\left(X_{(k)}\right)$ where $X$ are DAX returns and $q$ is risk neutral density. DAX returns are calculated on half year basis $X_{i}=\frac{X_{i}-X_{i-126}}{X_{i-126}}$ and then ordered to $X_{(k)}$. Corresponding ordered returns differences $X_{(k+1)}-X_{(k)}$ for years 2000, 2002 and 2004 are displayed in Figure 5.1.

Risk neutral density $q$ (see Figure 5.2) is estimated using Heston model (1993) calibrated on observed implied volatility surfaces with half year maturity. Fore more details on estimation of risk neutral density refer to Detlefsen, et al (2007).

Resulting $Z_{k}$ values are displayed in Figure 5.3. For each set of $Z_{k}$ an isotonic regression was constructed which represents $\max _{K \subset A(I, J)} \log \{p(Z, K)\}$ in equation (7). Numerous simulations showed that in order to compute maximum likelihood for restricted model 

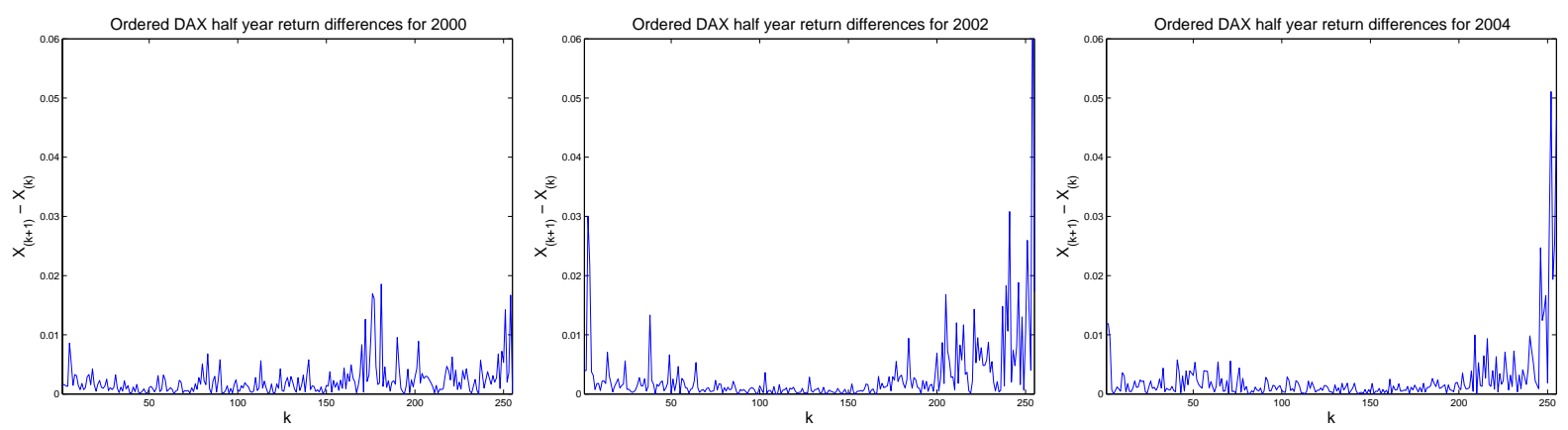

Figure 5.1: Half year ordered returns differences $X_{(k+1)}-X_{(k)}$ for years 2000, 2002 and 2004
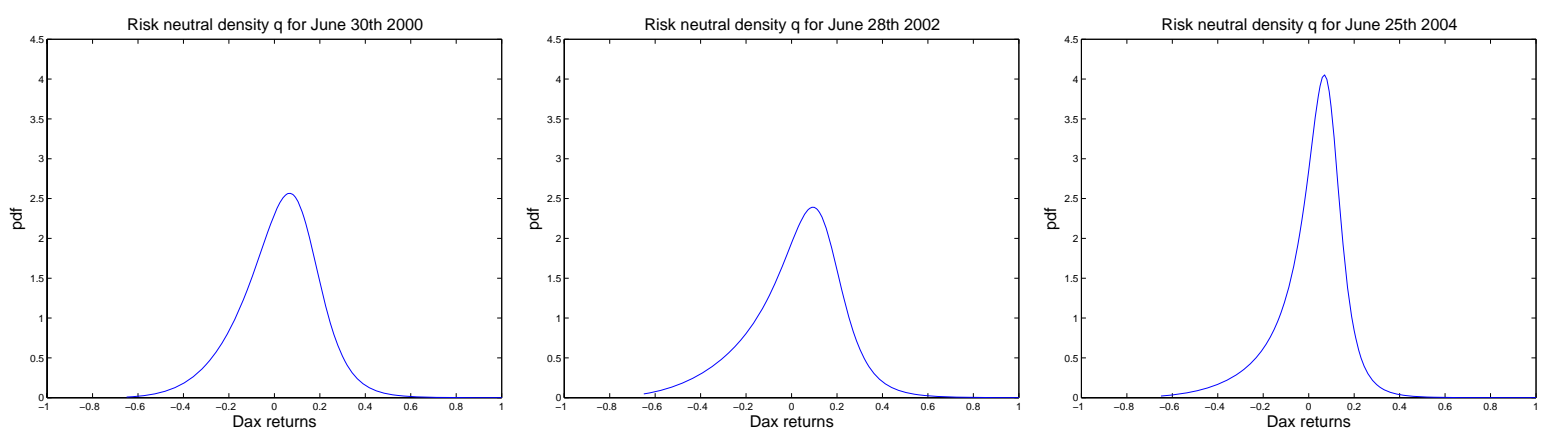

Figure 5.2: Estimated risk neutral densities $q$ for years 2000, 2002 and 2004

$\max _{K \subset A(I, J)} \log \{p(Z, K)\}$ we have to take isotonic regression over optimal parameters which maximize $\log \{p(Z, K)\}$ for all possible $K \cdot \max _{K} \log \{p(Z, K)\}$ is reached at $K_{k}=Z_{k}$ and equal to $-n-\sum_{k=1}^{n} \log \left(Z_{k}\right)$, so isotonic regression over observed $Z_{k}$ maximizes $\max _{K \subset A(I, J)} \log \{p(Z, K)\}$.

In order to take a final decision about the motonocity sequence of surfaces $M(I, I, b)$ and $V(I, J, b)$ has to be computed. $M$ and $V^{2}$ are mean and variance parameters of test statistics $\xi$ obtained via Monte Carlo simulations of $Z_{k}$ as iid standard exponential random variable. Each value of the matrixes $M$ and $V$ represent correspondingly mean and standard error of $\xi$ for a fixed parameter $b$ and interval $I, J$ and calculated as $\max _{K \subset A(I, J)} \log \{p(Z(I, J), K)\}-$ $\max _{K} \log \{p(Z(I, J), K)\}$. Matrix $M$ has non-positive values with maximum at $0, V$ is nonnegative. Both matrixes exist only for $J>I$, see Section 3.2 for details. Since surfaces $M$ and $V$ do not depend on observed data but only on the number of observations $n$ and block 

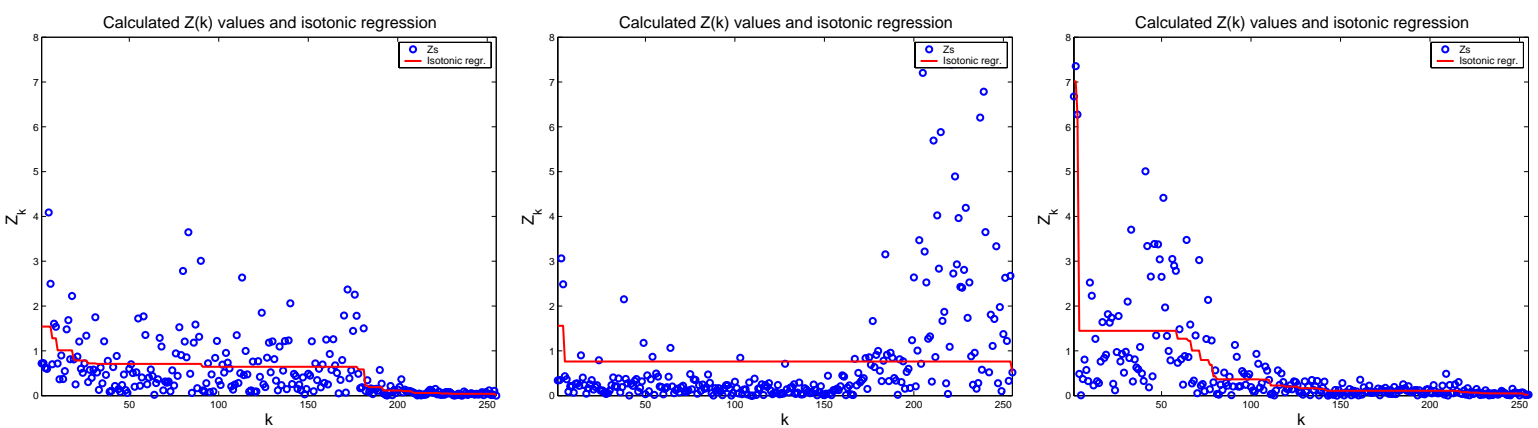

Figure 5.3: Calculated $Z_{k}$ for years 2000, 2002 and 2004

size $b$ they are computed once for all years. In Figure 5.4 corresponding surfaces $M(I, J)$ and $V(I, J)$ are plotted for $b=1, M$ is linear increasing in $I, J ; V$ is increasing in $I, J$ at square root speed.
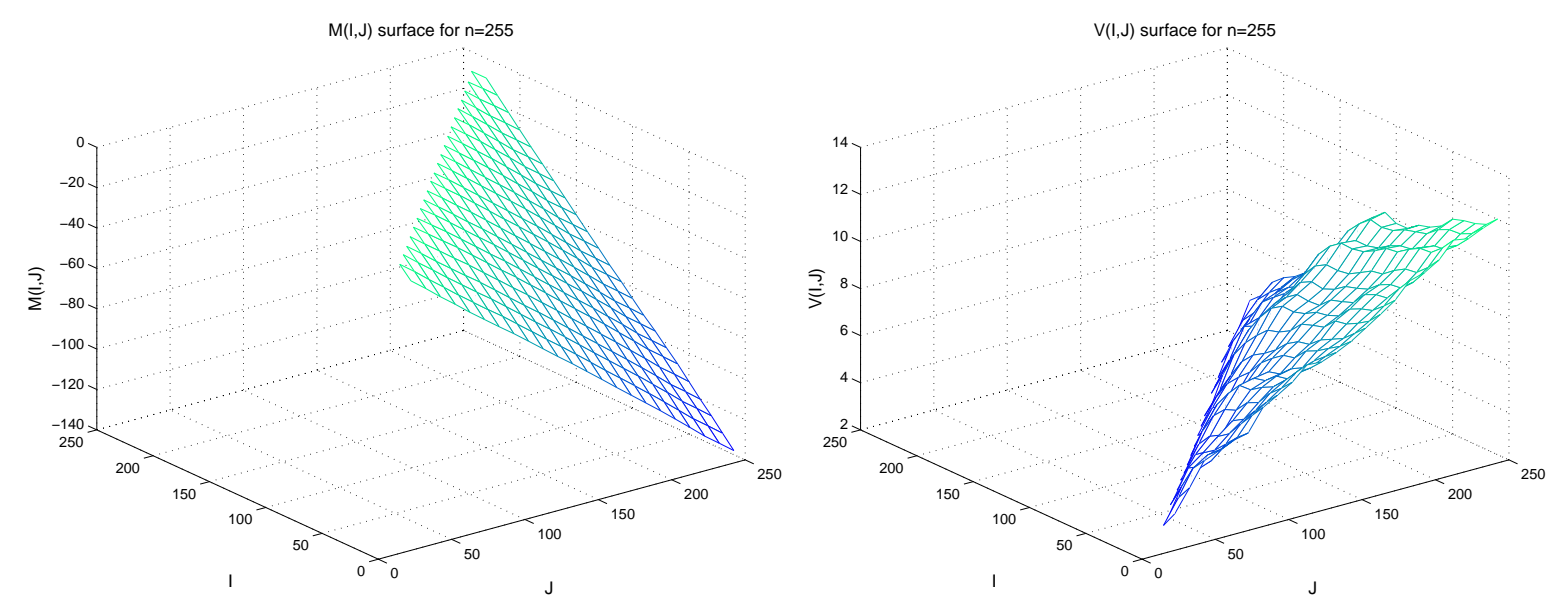

Figure 5.4: Surfaces $M$ and $V$ for 255 observations, $b=1$

Next important step is to calculate critical values $t_{\alpha, b}$ which are defined as a root to equation (10). This procedure is time consuming, but at the same time does not rely on data and has to be simulated once for a fixed number of observation $n$ and block size $b$. We use dichotomic method of iterative splitting intervals. In our analysis we start with intervals $[0.0,20.0]$ then calculate correponding $\alpha$ for the mean of the interval. Depending on calculated $\alpha$ one of two resulting intervals $[0.0,10.0]$ and $[10.0,20.0]$ is chosen. This procedure is repeated for selected 
interval until solution of required precision is found. Resulting critical values are presented in Figure 5.5. It can be seen that critical values are changing for different parameter $b$.

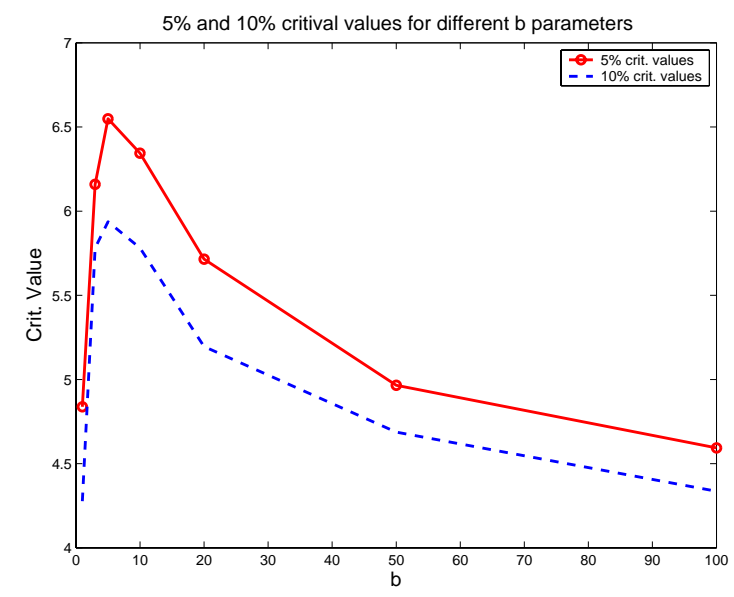

Figure 5.5: $5 \%$ and 10\% distribution over $b$

Finally testing surfaces $\xi(I, J, b)-M(I, J, b)+t_{0.05, b} \cdot V(I, J, b)$ for years 2000, 2002 and 2004 are produced. For fixed $b=50$ corresponding surfaces are presented in figure 5.6. They show the differences between simulated $5 \%$ threshold surface $M-t_{0.05,50} \cdot V$ calculated via Monte Carlo simulations and test statistics $\xi$ obtained from observed data in years 2000, 2002 and 2004. Hypothesis $H_{0}$ of motononic descreasing EPK is rejected at $5 \%$ significance level if test statistics $\xi$ is smaller than threshold value $M(I, J)-t_{0.05} V(I, J)$. For each interval $I, J$ where surface $\xi(I, J, 50)-M(I, J, 50)+t_{0.05,50} V(I, J, 50)$ is negative, a corresponding rejection interval is plotted in Figure 5.7. Summary of results for three years is presented in Table 5.1. In addition to accepted hypothesis, value of $\min _{I, J, b}\left\{\xi(I, J, b)-M(I, J, b)+t_{\alpha, b} \cdot V(I, J, b)\right\}$ is given in the table. By evaluating this values we can estimate the significance of accepted hypotheses. Test significantly rejectes monotone decreasing EPK in 2002 as well as can not reject strictly decreasing EPK in 2004 for 5\% and 10\% significance level. Situation in 2002 is on the verge: $H_{0}$ can not be rejected with $5 \%$ critical values, but rejected at $10 \%$ signifiance level. 

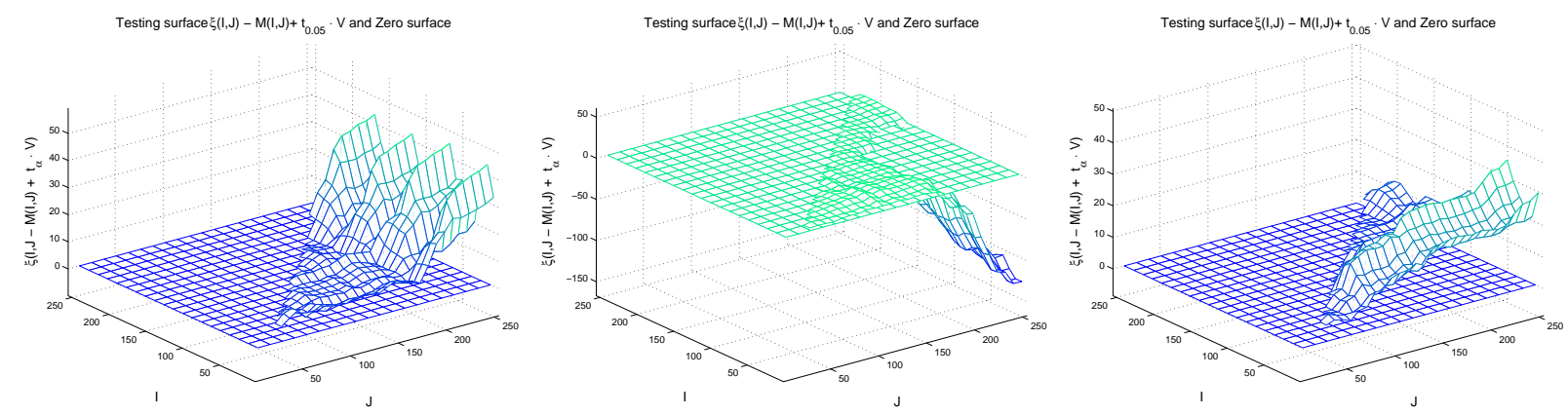

Figure 5.6: Surface $\xi(I, J, 50)-M(I, J, 50)-t_{0.05,50} \cdot V(I, J, 50)$ for years $2000,2002,2004$
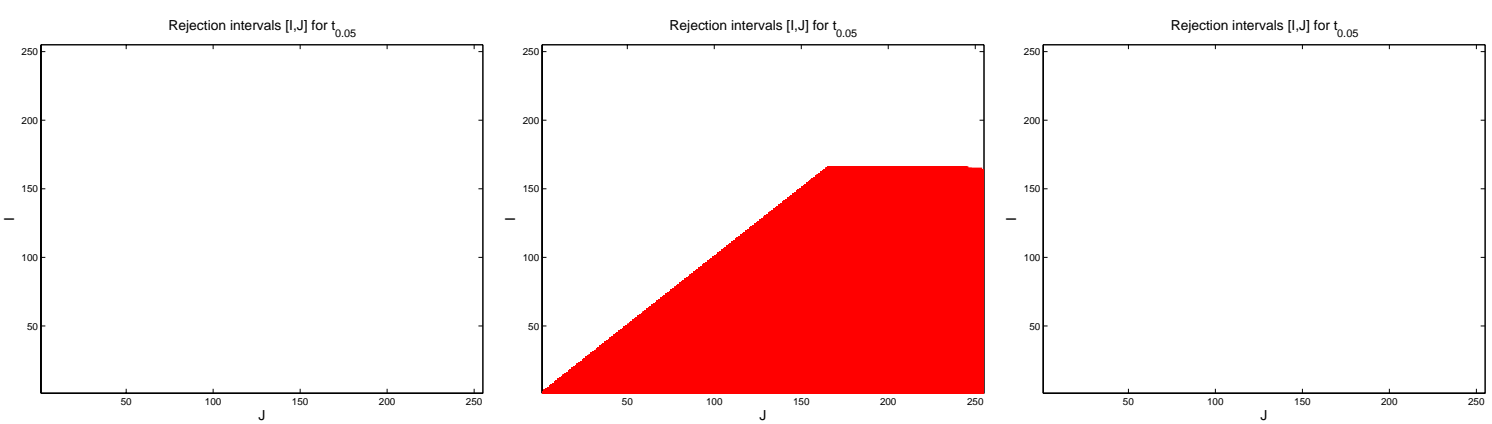

Figure 5.7: Rejection intervals $(I, J)$ for years 2000, 2002 and 2004, $b=50$

\begin{tabular}{llll}
\hline \hline Sign. level/Year of analysis & 2000 & 2002 & 2004 \\
\hline $5 \%$ Significance level & & & \\
$\min _{I, J, b}$ & 0.5437 & -133.78 & 3.7935 \\
Accepted $H_{0}$ & $H_{0}$ & $H_{1}$ & $H_{0}$ \\
\hline $10 \%$ Significance level & & & \\
$\min _{I, J, b}$ & -0.1840 & -134.42 & 3.1685 \\
Accepted $H_{1}$ & $H_{1}$ & $H_{1}$ & $H_{0}$ \\
\hline \hline
\end{tabular}

Table 5.1: Summary of results on monotonicity of EPK in 2000, 2002 and 2004. 


\section{Conclusion}

In this paper we describe the test that checks monotonicity of pricing kernels. By testing monotonicity of pricing kernel we can determine whether utility function is concave or not. A strictly decreasing pricing kernel corresponds to a concave utility function while nondecreasing EPK means that the utility function contains some non-concave regions.

Pricing kernels are constructed as a ratio of risk neutral density $q$ and subjective density $p$. Density $q$ is obtained from the derivative market and due to the large number of observations can be precisely estimated. $p$ is usually estimated from historical information, but due to scarcity of data is considered to be unknown. We therefore test the ratio of two densities $\frac{q}{p}$, where $q$ is given and $p$ is unknown. Using Pyke's theorem (see Pyke, R. (1965)) this problem is reduced to a simple exponential problem. The test itself is constructed on the basis of the likelihood ratio test for a fixed interval. By using the intersection of tests for different intervals we can expand it to the variant which is independent of intervals. In section 3.3 we introduce a block parameter, which allows a function to be monotone on intervals of size $b$. This innovation improves the power of the test by searching for the tradeoff between the shift and variance of test distribution function.

We investigated EPK for German DAX data for years 2000, 2002 and 2004. We found the evidence of non-concave utility function: $H_{0}$ hypothesis of monotone decreasing pricing kernel function was rejected at 5\% and 10\% significance level in 2002 and at $10 \%$ significance level monotonicity in 2000. This result is consistent with the work of Jackwerth, J. C. (2002) who observed partially negative risk aversion during the post crash period. For year 2004 a hypothesis of decreasing EPK could not be rejected at $5 \%$ as well as at $10 \%$ significance level. These findings also support the idea of Giacomini and Haerdle (2007) who wrote the the structure of pricing kernel may vary over time. 


\section{References}

Ait-Sahalia, Y. and Lo, A. (2000). Nonparametric risk management and implied risk aversion. Journal of Econometrics 94(12), 951.

Ait-Sahalia, Y., Yubo W., and Francis Y. (2001). Do option markets correctly price the probabilities of movement of the underlying asset? Journal of Econometrics 102(1), 67110.

Berger, R. L. (1982) Multiparameter hypothesis testing and acceptance sampling. Technometrics 24: 295-300.

Black, F. and Scholes, M. (1973) The pricing of options and corporate liabilities. Journal of Political Economy, 81: 637-659.

Cox, J. C., Jonathan E. I., and Stephen A. R. (1985). An Intertemporal General Equilibrium Model of Asset Prices. Econometrica, 363-384.

Detlefsen, Härdle, W. K. and Moro, R. M. (2007). Empirical Pricing Kernels and Investor Preferences, SFB 649 Discussion Paper 200\%-01\%.

Giacomini, E. and Härdle, W. (2007). Statistics of Risk Aversion, proceedings, 56th Session of the International Statistical Institute, Lisbon $200 \%$.

Giacomini, E., Handel, M. and Härdle, W. (2006). Time Dependent Relative Risk Aversion, proceedings, 9th Karlsruhe Econometrics Workshop 2006.

Jackwerth, J. C. (2002). Recovering Risk Aversion from Option Prices and Realized Returns, Review of Financial Studies, 13, 433-451.

Heston, S. (1993). A Closed-Form Solution for Options with Stochastic Volatility with Applications to Bond and Currency Options, Review of Financial Studies, 6, (2), 327-343.

Kahneman, D and Tversky, A. (1979). Prospect Theory: An Analysis of Decision under Risk, Econometrica, 47, March, 263-291. 
Merton, R. C. (1973). An Intertemporal Capital Asset Pricing Model, Econometrica, 41, (5), $867-88 \%$

Pyke, R. (1965 ). Spacings, J.R. Statistical Society, B 27, 395-436.

Proschan, F. and Pyke, R. (1967). Tests for Monotone Failure Rate, Fifth Berkeley Symposium, 3, 293-313.

Rosenberg, J. and Engle, R. (2002). Empirical Pricing Kernels, Journal of Financial Economics, 64, June, 341-372. 


\section{SFB 649 Discussion Paper Series 2008}

For a complete list of Discussion Papers published by the SFB 649, please visit http://sfb649. wiwi. hu-berlin.de.

001 "Testing Monotonicity of Pricing Kernels" by Yuri Golubev, Wolfgang Härdle and Roman Timonfeev, January 2008.

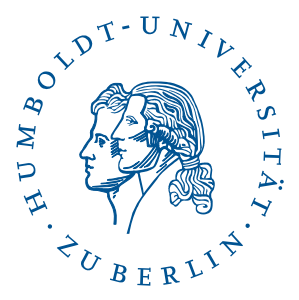

